\title{
Nash Bargaining over MIMO Interference Systems
}

\author{
Zengmao Chen ${ }^{\dagger}$, Sergiy A. Vorobyov ${ }^{\dagger \dagger}$, Cheng-Xiang Wang ${ }^{\dagger}$, and John Thompson ${ }^{\dagger \dagger}$

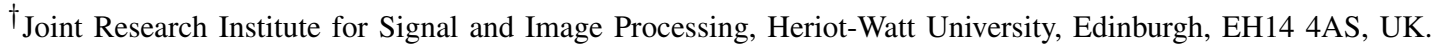 \\ ${ }^{\dagger \dagger}$ Department of Electrical and Computer Engineering, University of Alberta, Edmonton, AB, T6G 2V4, Canada. \\ ${ }^{\dagger \dagger}$ Joint Research Institute for Signal and Image Processing, University of Edinburgh, Edinburgh, EH9 3JL, UK. \\ Email: zc34@hw.ac.uk, vorobyov@ece.ualberta.ca, cheng-xiang.wang@hw.ac.uk, john.thompson@ed.ac.uk
}

\begin{abstract}
In this paper, the source covariance matrices of multiple-input multiple-output (MIMO) interference channels (IFCs) are investigated from a game-theoretic perspective. It is proved that the requirement of sufficiently small interference-tonoise ratio (INR) is the sufficient condition for the uniqueness of the Nash bargaining (NB) solution. The structure of the source covariance matrices, which constitute the feasible set of NB solution, is analyzed by comparing them with the covariance matrices leading to the Nash equilibrium (NE). The existence of the NB solution and concavity of the rate product for MIMO IFCs are also studied.
\end{abstract}

Index Terms - MIMO interference channel, Nash bargaining, Nash equilibrium, source covariance matrix, game theory.

\section{INTRODUCTION}

With the philosophy of sharing the available spectrum with either peer networks or legacy systems, dynamic spectrum access (DSA) [1] holds the potential to significantly improve the spectrum utilization. The newly emerged cognitive radio technology [2] is one of its promising implementations, where all secondary users try to share the spectrum holes and operate in an interference channel (IFC). Besides traditional information-theoretic approaches, there has recently been a new research trend to study IFCs from a game-theoretic perspective.

Various IFCs have been investigated in the literature, where the Nash equilibrium (NE) and Nash bargaining (NB) were applied to interference games stemming from these IFCs. The NB was theoretically examined for single-input single-output (SISO) and multiple-input single-output (MISO) IFCs in [3] and [4] in terms of its existence, respectively. The practical algorithms finding the NB solution for SISO and MISO IFCs were proposed in [5] and [6], respectively. The NB over IFCs was extended to the MIMO case in [7], where a practical suboptimal algorithm for finding the NB solution was designed by exploring the gradient projection method [8]. To the best of the authors' knowledge, there have been no research attempts to theoretically study the NB in MIMO IFCs in terms of its uniqueness and existence, which motivates the research in this paper.

In this paper, we fill this gap by deriving a sufficient condition for the uniqueness of the NB solution in MIMO interference systems. The feasible NB set is also analyzed by studying the structure of the source covariance matrices. Finally, the existence of the NB solution and concavity of the rate product for MIMO IFCs are investigated in terms of simulations.

\section{System Model AND PROBlem Formulation}

\section{A. System Model}

Consider an $L$-link MIMO interference system with $L$ transmitters and $L$ corresponding receivers, where the transmitter and receiver for each link are equipped with $N_{t}$ and $N_{r}$ antennas, respectively. The $N_{r} \times 1$ complex base-band signal vector received by link $i(i=1,2, \ldots, L)$ can be written as

$$
\mathbf{y}_{i}=\sqrt{\rho_{i}} \mathbf{H}_{i i} \mathbf{x}_{i}+\sum_{j=1, j \neq i}^{L} \sqrt{\eta_{i j}} \mathbf{H}_{i j} \mathbf{x}_{j}+\mathbf{n}_{i}
$$

where $\rho_{i}$ is the normalized signal-to-noise ratio (SNR) for link $i ; \eta_{i j}$ is the normalized interference-to-noise radio (INR) from transmitter $j$ to receiver $i ; \mathbf{H}_{i i}$ and $\mathbf{H}_{i j}$ are the $N_{r} \times N_{t}$ channel matrices from transmitter $i$ and transmitter $j$ to receiver $i$, respectively; $\mathbf{x}_{i}$ is the $N_{t} \times 1$ transmitted signal vector for link $i$, and $\mathbf{n}_{i}$ is the $N_{r} \times 1$ independently identically distributed (i.i.d.) additive white Gaussian noise (AWGN) vector perceived by link $i$ with zero mean and identity covariance matrix $E\left[\mathbf{n}_{i} \mathbf{n}_{i}^{H}\right]=\mathbf{I}, E[\cdot]$ is the expectation operator, $(\cdot)^{H}$ denotes the Hermitian transpose operation, and $\mathbf{I}$ is the identity matrix.

We assume that: 1) each transmitter/receiver transmits/receives symbols independently; 2) the co-channel interference from other links is unknown and treated as noise (i.e., no interference cancellation (IC) techiniques are employed by receivers); 3) the channel varies sufficiently slow and can be considered as time invariant during the period of each symbol transmission.

The mutual information for user $i$ can be expressed as [9]

$$
I_{i}(\mathbf{Q})=\log _{2} \operatorname{det}\left(\mathbf{I}+\rho_{i} \mathbf{Q}_{i} \mathbf{H}_{i i}^{H} \mathbf{R}_{-i}^{-1} \mathbf{H}_{i i}\right) \quad i=1, \ldots, L
$$

where $\mathbf{Q}_{i}=E\left[\mathbf{x}_{i} \mathbf{x}_{i}^{H}\right]$ is the Hermitian positive semi-definite (PSD) source covariance matrix of the input signal vector for link $i$ and $\mathbf{R}_{-i}=\mathbf{I}+\sum_{j=1, j \neq i}^{L} \eta_{i j} \mathbf{H}_{i j} \mathbf{Q}_{j} \mathbf{H}_{i j}^{H}$ is the covariance matrix of the interference-plus-noise received by receiver $i$. We define $\mathbf{Q} \triangleq\left(\mathbf{Q}_{1}, \ldots, \mathbf{Q}_{L}\right)$ as a set of source covariance matrices. Since the transmission of each user is power limited, the following trace constraint applies to $\mathbf{Q}_{i}$

$$
\operatorname{tr}\left(\mathbf{Q}_{i}\right) \leq p_{i}
$$

where $\operatorname{tr}(\cdot)$ denotes the trace of a matrix. We also assume that each transmitter $i$ has the full knowledge of channel, 
SNR, INR and the source covariance matrices of all the other transmitters. We optimize the mutual information of each user by choosing the source covariance matrix $\mathbf{Q}_{i}$ under the trace constraint (3).

\section{B. Game Theoretic Formulation - NE}

The MIMO interference system delineated above can be modeled as a MIMO interference game, whose players are the users in the MIMO interference system. The mutual information of each user is taken as the utility of the corresponding player. The source covariance matrix of each user forms the strategy space of each player.

In a competitive game, as the name suggests, all the players compete with each other selfishly and rationally. Players neither communicate nor cooperate with each other prior to the game. A steady state in a competitive game where each player can not improve its utility by unilaterally changing its own strategy is called the NE. For a MIMO interference game, its NE can be mathematically expressed as

$$
I_{i}\left(\mathbf{Q}_{i}^{*}, \mathbf{Q}_{-i}^{*}\right) \geq I_{i}\left(\mathbf{Q}_{i}, \mathbf{Q}_{-i}^{*}\right) \quad i=1, \ldots, L
$$

where $\mathbf{Q}_{i}^{*}, \mathbf{Q}_{-i}^{*}$ are the source covariance matrices of $\mathrm{NE}$ for user $i$ and for all the other users except $i$, respectively.

The source covariance matrix of each player leading to NE can be found via iterative water filling (IWF) [10]

$$
\mathbf{Q}_{i}^{*}=\mathbf{U}_{i}\left(\mu_{i} \mathbf{I}-\mathbf{D}_{i}^{-1}\right)^{+} \mathbf{U}_{i}^{H}
$$

where $\mathbf{U}_{i} \mathbf{D}_{i} \mathbf{U}_{i}^{H}=\mathbf{H}_{i i}^{H} \mathbf{R}_{-i}^{-1} \mathbf{H}_{i i}$ is the eigendecomposition of $\mathbf{H}_{i i}^{H} \mathbf{R}_{-i}^{-1} \mathbf{H}_{i i}, \mathbf{U}_{i}$ is the unitary matrix of eginevectors, $\mathbf{D}_{i}$ is a diagonal matrix of eigenvalues, and $\mu_{i}$ denotes the power level given by IWF.

\section{Game Theoretic Formulation - NB}

Generally, NE is not optimal from the system point of view, due to its competitive and selfish nature. Whereas, in a cooperative game, all the players negotiate with each other prior to the game, which usually results in utility improvement. There exist many cooperative game-theoretic approaches, here we restrict our attention to the NB [11].

In this case, each player $i$ has a set of strategies (bargaining set) which it may implement. In the context of the MIMO interference games, the bargaining set for user $i$ can be expressed as

$$
S=\left\{\mathbf{Q}_{i} \mid \mathbf{Q}_{i} \text { is PSD, } \operatorname{tr}\left(\mathbf{Q}_{i}\right) \leq p_{i} \text {, and } I_{i}(\mathbf{Q})>I_{i}^{N E}, i=1, \ldots, L\right\} \text {. }
$$

It also has a disagreement point, which is defined as the state that players resort to when the cooperation fails. Usually, NE is taken as the disagreement point.

Stated in the mathematical form, the NB for the MIMO interference game can be written as the following optimization problem

$$
\begin{array}{ll}
\max _{\mathbf{Q}} \prod_{i=1}^{L}\left(I_{i}(\mathbf{Q})-I_{i}^{N E}\right) \\
\text { subject to } \quad \operatorname{tr}\left(\mathbf{Q}_{i}\right) \leq p_{i} \quad i=1, \ldots, L \\
& I_{i}(\mathbf{Q})>I_{i}^{N E} \quad i=1, \ldots, L .
\end{array}
$$

It is worth noting that the $\mathrm{NB}$ corresponds to proportional fairness, and Nash product $\prod_{i=1}^{L}\left(I_{i}(\mathbf{Q})-I_{i}^{N E}\right)$ is converted to the rate product of all links when $I_{i}^{N E}=0$.

In the sequel, we first investigate the uniqueness of the NB solution and then briefly examine the feasible bargaining set and the existence of NB solution.

\section{UNIQUENESS OF NB SOLUTION}

Proposition: The INR $\eta_{i j}$ being sufficiently small is the sufficient condition for the uniqueness of the NB solution of the MIMO interference systems.

Proof: It can be shown that the solution for (7) is identical to that of the following optimization problem

$$
\begin{aligned}
\max _{\mathbf{Q}} \ln \left(\ln ^{L} 2 \cdot \prod_{i=1}^{L}\left(I_{i}(\mathbf{Q})-I_{i}^{N E}\right)\right) \\
\text { subject to } \quad \operatorname{tr}\left(\mathbf{Q}_{i}\right)-p_{i} \leq 0 \quad i=1, \ldots, L \\
\\
I_{i}^{N E}-I_{i}(\mathbf{Q})<0 \quad i=1, \ldots, L .
\end{aligned}
$$

The optimization problem (8) is a convex one if and only if its objective function is concave and its constraint set is convex [12]. When (8) is a convex optimization problem, there exists at most one solution maximizing the objective function of (8), which is the sufficient condition for the uniqueness of the NB solution.

\section{A. Concavity of Objective Function}

A function $g(x)$ is concave if and only if 1) $f(t)=g\left(t x_{1}+\right.$ $\left.(1-t) x_{2}\right), 0 \leq t \leq 1$ is a concave function of $t$ for any feasible $x_{1}$ and $x_{2}$, which is equivalent to $d^{2} f(t) / d t^{2} \leq 0 ; 2$ ) the domain of $g(x)$ is convex [12].

We consider the convex combination of two different feasible solutions of (8): $\left(\mathbf{Z}_{1}, \ldots, \mathbf{Z}_{L}\right)$ and $\left(\mathbf{X}_{1}, \ldots, \mathbf{X}_{L}\right)$, that is

$$
\begin{aligned}
\mathbf{Q}(t) & =\left(\mathbf{Q}_{1}, \ldots, \mathbf{Q}_{L}\right) \\
& =(1-t)\left(\mathbf{X}_{1}, \ldots, \mathbf{X}_{L}\right)+t\left(\mathbf{Z}_{1}, \ldots, \mathbf{Z}_{L}\right) \\
& =\left(\mathbf{X}_{1}, \ldots, \mathbf{X}_{L}\right)+t\left(\mathbf{Z}_{1}-\mathbf{X}_{1}, \ldots, \mathbf{Z}_{L}-\mathbf{X}_{L}\right) \\
& =\left(\mathbf{X}_{1}, \ldots, \mathbf{X}_{L}\right)+t\left(\mathbf{Y}_{1}, \ldots, \mathbf{Y}_{L}\right)
\end{aligned}
$$

where $0 \leq t \leq 1$. Let us define

$$
\begin{aligned}
f(t) & =\ln \left(\ln ^{L} 2 \cdot \prod_{i=1}^{L}\left(I_{i}(\mathbf{Q}(t))-I_{i}^{N E}\right)\right) \\
& =\sum_{i=1}^{L} \ln \left(\ln \frac{\operatorname{det}\left(\mathbf{R}_{-i}+\rho_{i} \mathbf{H}_{i i} \mathbf{Q}_{i} \mathbf{H}_{i i}^{H}\right)}{\operatorname{det}\left(\mathbf{R}_{-i}\right)}-\ln 2 \cdot I_{i}^{N E}\right)
\end{aligned}
$$

and

$$
T_{i}=\ln \frac{\operatorname{det}\left(\mathbf{R}_{-i}+\rho_{i} \mathbf{H}_{i i} \mathbf{Q}_{i} \mathbf{H}_{i i}^{H}\right)}{\operatorname{det}\left(\mathbf{R}_{-i}\right)}-\ln 2 \cdot I_{i}^{N E} .
$$

By applying the well known properties of matrix differential calculus [13], we have

$$
\begin{gathered}
f^{\prime}(t)=\sum_{i=1}^{L} \frac{1}{T_{i}} \cdot\left[\operatorname{tr}\left(\left(\mathbf{R}_{-i}+\rho_{i} \mathbf{H}_{i i} \mathbf{Q}_{i} \mathbf{H}_{i i}^{H}\right)^{-1}\left(\frac{d \mathbf{R}_{-i}}{d t}+\rho_{i} \mathbf{H}_{i i} \mathbf{Y}_{i} \mathbf{H}_{i i}^{H}\right)\right)\right. \\
\left.-\operatorname{tr}\left(\mathbf{R}_{-i}^{-1} \frac{d \mathbf{R}_{-i}}{T i}\right)\right] .
\end{gathered}
$$


Let $\mathbf{M}_{i}=\rho_{i} \mathbf{H}_{i i} \mathbf{Q}_{i} \mathbf{H}_{i i}^{H}$ and $\mathbf{N}_{i}=\rho_{i} \mathbf{H}_{i i} \mathbf{Y}_{i} \mathbf{H}_{i i}^{H}$, then $f^{\prime \prime}(t)$ can be expressed as

$$
f^{\prime \prime}(t)=\sum_{i=1}^{L} \alpha+\beta+\gamma
$$

$\alpha=\frac{\operatorname{tr}\left(-\left(\mathbf{R}_{-i}+\mathbf{M}_{i}\right)^{-1}\left(\frac{d \mathbf{R}_{-i}}{d t}+\mathbf{N}_{i}\right)\left(\mathbf{R}_{-i}+\mathbf{M}_{i}\right)^{-1}\left(\frac{d \mathbf{R}_{-i}}{d t}+\mathbf{N}_{i}\right)\right)}{T_{i}}$

$$
\beta=\frac{\operatorname{tr}\left(\mathbf{R}_{-i}^{-1} \frac{d \mathbf{R}_{-i}}{d t} \mathbf{R}_{-i}^{-1} \frac{d \mathbf{R}_{-i}}{d t}\right)}{T_{i}}
$$

$\gamma=-\frac{\left[\operatorname{tr}\left(\left(\mathbf{R}_{-i}+\mathbf{M}_{i}\right)^{-1}\left(\frac{d \mathbf{R}_{-i}}{d t}+\mathbf{N}_{i}\right)\right)-\operatorname{tr}\left(\mathbf{R}_{-i}^{-1} \frac{d \mathbf{R}_{-i}}{d t}\right)\right]^{2}}{T_{i}^{2}}$

Let $\mathbf{A}_{i}=\left(\mathbf{R}_{-i}+\mathbf{M}_{i}\right)^{-1}$ and $\mathbf{B}_{i}=\frac{d \mathbf{R}_{-i}}{d t}+\mathbf{N}_{i}$. Since $\mathbf{A}_{i}$ is PSD, there exists a matrix $\mathbf{C}_{i}$ such that $\mathbf{A}_{i}=\mathbf{C}_{i} \mathbf{C}_{i}^{H}$. Thus, the numerator of (14) can be written as

$$
\begin{aligned}
& \operatorname{tr}\left(-\mathbf{A}_{i} \mathbf{B}_{i} \mathbf{A}_{i} \mathbf{B}_{i}\right) \\
& =-\operatorname{tr}\left(\mathbf{C}_{i} \mathbf{C}_{i}^{H} \mathbf{B}_{i} \mathbf{C}_{i} \mathbf{C}_{i}^{H} \mathbf{B}_{i}\right) \\
& =-\operatorname{tr}\left(\mathbf{C}_{i}^{H} \mathbf{B}_{i} \mathbf{C}_{i} \mathbf{C}_{i}^{H} \mathbf{B}_{i} \mathbf{C}_{i}\right) \\
& =-\operatorname{tr}\left(\left(\mathbf{C}_{i}^{H} \mathbf{B}_{i} \mathbf{C}_{i}\right)\left(\mathbf{C}_{i}^{H} \mathbf{B}_{i} \mathbf{C}_{i}\right)^{H}\right) \leq 0
\end{aligned}
$$

The last equality in (17) is obtained based on the fact that $\mathbf{B}_{i}$ is Hermitian, and $\left(\mathbf{C}_{i}^{H} \mathbf{B}_{i} \mathbf{C}_{i}\right)\left(\mathbf{C}_{i}^{H} \mathbf{B}_{i} \mathbf{C}_{i}\right)^{H}$ is PSD. If the bargaining set $S$ is not empty, then $T_{i}>0$. Thus, $\alpha$ is not positive.

In (15), if the INR $\eta_{i j}$ is sufficiently small, then $\mathbf{R}_{-i}$ approaches $\mathbf{I}$, as the noise dominates the interference; $\frac{d \mathbf{R}_{-i}}{d t}=\sum_{j \neq i} \eta_{i j} \mathbf{H}_{i j} \mathbf{Y}_{i j} \mathbf{H}_{i j}^{H}$ is proportional to $\eta_{i j}$ and $\left(\mathbf{R}_{-i}^{-1} \frac{d \mathbf{R}_{-i}}{d t} \mathbf{R}_{-i}^{-1} \frac{d \mathbf{R}_{-i}}{d t}\right)$ is proportional to $\eta_{i j}^{2}$; while in (14) $\left(\mathbf{R}_{-i}+\mathbf{M}_{i}\right)^{-1}$ and $\left(\frac{d \mathbf{R}_{-i}}{d t}+\mathbf{N}_{i}\right)$ are dominated by $\left(\mathbf{I}+\mathbf{M}_{i}\right)^{-1}$ and $\mathbf{N}_{i}$ respectively, i.e., $\eta_{i j}$ has little impact on (14). Hence, $\beta$ can be ignored compared to $\alpha$ when $\eta_{i j}$ is sufficiently small.

It can be seen that $\gamma$ is not positive. Therefore, when $\eta_{i j}$ is sufficiently small, $f^{\prime \prime}(t) \leq 0$.

The domain of the objection function in (8) is $\left\{\mathbf{Q}_{i} \mid \mathbf{Q}_{i}\right.$ is PSD, $\left.\operatorname{tr}\left(\mathbf{Q}_{i}\right)-p_{i} \leq 0, i=1, \ldots, L\right\}$, which is obviously convex. Therefore, the objective function is concave.

\section{B. Convexity of Constraint Set}

The constraint set of (8) is identical to the bargain set (6). Specifically, it can be rewritten as

$$
\begin{aligned}
S= & \left\{\mathbf{Q}_{i} \mid \mathbf{Q}_{i} \text { is PSD }, \operatorname{tr}\left(\mathbf{Q}_{i}\right)-p_{i} \leq 0, i=1, \ldots, L\right\} \cap \\
& \left\{\mathbf{Q}_{i} \mid-I_{i}(\mathbf{Q})+I_{i}^{N E} \leq 0, i=1, \ldots, L\right\} \\
= & S_{1} \cap S_{2}
\end{aligned}
$$

It is easy to establish the convexity of the subset $S_{1}$, but the convexity of subset $S_{2}$ is not obvious.

Let us define $h(t)=-I_{i}(\mathbf{Q}(t))+I_{i}^{N E}$. Adopting the same methodology as in section III-A, we have

$h^{\prime \prime}(t)=\operatorname{tr}\left(\left(\mathbf{R}_{-i}+\mathbf{M}_{i}\right)^{-1}\left(\frac{d \mathbf{R}_{-i}}{d t}+\mathbf{N}_{i}\right)\left(\mathbf{R}_{-i}+\mathbf{M}_{i}\right)^{-1}\left(\frac{d \mathbf{R}_{-i}}{d t}+\mathbf{N}_{i}\right)\right.$

$$
\left.-\mathbf{R}_{-i}^{-1} \frac{d \mathbf{R}_{-i}}{d t} \mathbf{R}_{-i}^{-1} \frac{d \mathbf{R}_{-i}}{d t}\right)
$$

The sufficiently small $\eta_{i j}$ imposes little impact on the first term inside the trace operator in (19), whereas its second term can be ignored. Thus, similar to (17), when $\eta_{i j}$ is sufficiently small, (19) can be rewritten as

$$
\begin{aligned}
h^{\prime \prime}(t) & \approx \operatorname{tr}\left(\left(\mathbf{R}_{-i}+\mathbf{M}_{i}\right)^{-1}\left(\frac{d \mathbf{R}_{-i}}{d t}+\mathbf{N}_{i}\right)\left(\mathbf{R}_{-i}+\mathbf{M}_{i}\right)^{-1}\left(\frac{d \mathbf{R}_{-i}}{d t}+\mathbf{N}_{i}\right)\right) \\
& =\operatorname{tr}\left(\left(\mathbf{C}_{i}^{H} \mathbf{B}_{i} \mathbf{C}_{i}\right)\left(\mathbf{C}_{i}^{H} \mathbf{B}_{i} \mathbf{C}_{i}\right)^{H}\right) \geq 0
\end{aligned}
$$

which implies that when $\eta_{i j}$ is sufficiently small, $h(t)$ is convex [12]. Consequently, the subset $S_{2}$ and then the constraint set $S$ are both convex.

As we can see, the condition that $\eta_{i j}$ is sufficiently small is sufficient for both the concavity of the objective function in (8) and the convexity of its constraint set. Therefore, the above proposition has been proved.

For the proportional fairness, where Nash product corresponds to the rate product, it is easy to infer that the INR $\eta_{i j}$ being sufficiently small is also the sufficient condition for the concavity of the rate product of the MIMO interference systems.

\section{Existence And Feasible NB Set}

As stated in section II, in MIMO IFCs, the NE is a steady state where each user best responds other users in term of mutual information. However, the NE is not Pareto efficient, i.e., it is usually below the rate boundary. If all the links simultaneously deviate from the NE and agree to adopt different strategies (i.e., source covariance matrices), this may result in simultaneous rate improvement for all links. In this case, the NB solution will exist.

The existence of the NB solution highly depends on the underlying MIMO interference channel, including its channel matrix, SNR and INR. It is difficult to find the closedform expression specifying when the NB solution exists. The general characteristics of the feasible utility set have been studied under an axiomatic framework in [14]. In this section, this problem will be investigated by analyzing the structure of the source covariance matrices forming the feasible bargaining set of the NB.

The source covariance matrix of each user is Hermitian PSD, thus it can be decomposed as

$$
\mathbf{Q}_{i}=\mathbf{V}_{i} \Lambda \mathbf{V}_{i}^{H}
$$

where $\mathbf{V}_{i}$ is an unitary matrix of eigenvectors of $\mathbf{Q}_{i}$ and $\boldsymbol{\Lambda}$ is the diagonal matrix of the corresponding eigenvalues. The source covariance matrices in the feasible set (6) can be considered as evolving from source covariance matrices of the NE (5). The evolution can be performed in two different ways:

1) changing the diagonal matrix $\left(\mu_{i} \mathbf{I}-\mathbf{D}_{i}^{-1}\right)^{+}$of (5);

2) modifying the unitary matrix $\mathbf{U}_{i}$ of (5).

The feasible set $S$ can be comprised of source covariance matrices of the following types:

I. $\mathbf{Q}_{i}$ has the same unitary matrix, but different diagonal matrix from $\mathbf{Q}_{i}^{*}$. This is also referred to as stream control [15], i.e., the number of the independent input 
data streams for each link is limited. This approach can be interpreted as selectively shutting down some transmission antennas, which create more interference to other links compared to the desired signal power generated for their target receivers.

II. $\mathbf{Q}_{i}$ has different unitary matrices, but the same diagonal matrix as $\mathbf{Q}_{i}^{*}$. This corresponds to the power control when all the effective links are the same, but their emitting directions are different from those of NE.

\section{Simulation Results}

\section{A. Feasible Set of $N B$}

Consider a 2-link MIMO interference system with $\mathbf{H}_{11}=$ $\operatorname{diag}(1.8,1), \mathbf{H}_{22}=\operatorname{diag}(1,1.8), \mathbf{H}_{12}=\mathbf{H}_{21}=\operatorname{diag}(1,1), \rho_{1}=$ $\rho_{2}=20, \eta_{12}=\eta_{21}=14$ and $p_{1}=p_{2}=1$. Its maximum achievable rate, the NE and NB solution are shown in Fig. 1. The source covariance matrices of the NE obtained by IWF are $\mathbf{Q}_{1}^{N E}=\operatorname{diag}(0.25,0.75)$, and $\mathbf{Q}_{2}^{N E}=\operatorname{diag}(0.75,0.25)$. The source covariance matrices resulting in NB are $\mathbf{Q}_{1}^{N B}=$ $\operatorname{diag}(1,0), \mathbf{Q}_{2}^{N B}=\operatorname{diag}(0,1)$. As we can see, $\mathbf{Q}_{i}^{N B}$ has the structure of Type I as mentioned in the previous section, with the same unitary matrix but different diagonal matrix from $\mathbf{Q}_{i}^{N E}$.

Consider another 2-link MIMO system with $\mathbf{H}_{11}=\mathbf{H}_{22}=$ $\left(\begin{array}{ll}2 & 2 \\ 1 & 1\end{array}\right), \mathbf{H}_{12}=\mathbf{H}_{21}=\left(\begin{array}{ll}1 & 0 \\ 0 & 1\end{array}\right), \rho_{1}=\rho_{2}=20, \eta_{12}=\eta_{21}=60$ and $p_{1}=p_{2}=1$. Fig. 2 demonstrates its maximum achievable rate, the NE and NB solution. The source covariance matrices of the NE are $\mathbf{Q}_{1}^{N E}=\mathbf{Q}_{2}^{N E}=\left(\begin{array}{ll}0.50 & 0.50 \\ 0.50 & 0.50\end{array}\right)=\left(\begin{array}{rr}-0.71 & 0.71 \\ 0.71 & 0.71\end{array}\right)$ $\cdot\left(\begin{array}{ll}0 & 0 \\ 0 & 1\end{array}\right) \cdot\left(\begin{array}{rr}-0.71 & 0.71 \\ 0.71 & 0.71\end{array}\right)$. Whereas, the source covariance matrices leading to the NB solution are $\mathbf{Q}_{1}^{N B}=\mathbf{Q}_{2}^{N B}=$ $\left(\begin{array}{ll}0.02 & 0.15 \\ 0.15 & 0.98\end{array}\right)=\left(\begin{array}{rr}-0.99 & 0.16 \\ 0.16 & 0.99\end{array}\right) \cdot\left(\begin{array}{ll}0 & 0 \\ 0 & 1\end{array}\right) \cdot\left(\begin{array}{rr}-0.99 & 0.16 \\ 0.16 & 0.99\end{array}\right)$. In this scenario, $\mathbf{Q}_{i}^{N B}$ has the structure of Type II, i.e., they have the same diagonal matrices but different unitary matrices. Alternatively, the source covariance matrix can be interpreted as precoding. And the corresponding precoding matrices for its NB and NE are $\mathbf{P}_{i}^{N B}=\left(\begin{array}{ll}0 & 0.16 \\ 0 & 0.99\end{array}\right), \mathbf{P}_{i}^{N E}=\left(\begin{array}{ll}0 & 0.71 \\ 0 & 0.71\end{array}\right)$.

\section{B. The SNR and INR Impact and Existence of NB Solution}

Fig. 3 depicts the SNR and INR impact on NE and NB solution. The channel realization is the same as that used for obtaining Fig.1. As we can see from Fig. 3, both NE and NB solution increase as SNR increases. In Fig. 4, the NB solution remains unchanging in the INR range where NB solution exists. This is due to the fact that the NB in this case adopts stream control, i.e., only one stream is transmitting for each user, which eventually converts the MIMO IFC into an interference-free system.

Fig. 5 presents the existence of NB solution at different values of SNR and INR. The symbol ' $'$ ' represents the fact that the NB solution exists at the corresponding SNR and INR. In this MIMO IFC, the NB solution exists only when its INR is smaller than the SNR. The rate of the links can be further improved by changing their source covariance matrices. From Fig. 4 and 5, it can be also seen that the NB solution does not exist when the INR is extremely small or large. This is due to the fact that the NE converges to the NB solution in these INR regions.

\section{Concavity of Rate Product}

Here, we examine the convexity of the rate product by simulations. Let us assume that the channel matrices are same as those used to obtain Fig. 1. We choose $t=0.5$. $\left(\mathbf{Z}_{1}, \mathbf{Z}_{2}\right)$ and $\left(\mathbf{X}_{1}, \mathbf{X}_{2}\right)$ are covariance matrices whose corresponding rates are in the rate boundary, and their rates for link 1 are $I_{1}^{N B}$ and $I_{1}^{N B} / 2$, respectively. Fig. 6 depicts the value of $g(t)=f^{\prime \prime}(t)$.It can be seen that: 1) The value of $g(t)$ almost solely depends on INR, i.e., INR has more impact on the concavity of rate product than SNR; 2) When the INR is sufficiently small, the rate product is concave (i.e., $g(t) \leq 0$ ). This result supports our findings in section III.

\section{Conclusions}

In this paper, the sufficient condition for the uniqueness of the NB solution in MIMO IFCs has been derived. The existence of NB solution and the structure of source covariance matrices constituting the feasible NB set have been analyzed. The simulation results have demonstrated the impact of the SNR and INR on the NE, NB and the existence of NB solution. These results also have shown the concavity of the rate product, which supports our theoretical findings.

There are still several issues deserving further investigation. First, the sufficient condition for the uniqueness of the NB solution is desirable to be given in a more accurate and quantitative form. Second, the study of the structure of the feasible NB set enquires using a heuristic approach to find the NB solution, which is computationally complex. Thus, an algorithm with less complexity is of interest. Third, we studied the pure strategies of the cooperative MIMO interference game without considering any multiplexing scheme. Further studies could be extended to those with multiplexing schemes.

\section{ACKNOWLEDGEMENTS}

We acknowledge the support from the Scottish Funding Council for the Joint Research Institute in Signal and Image Processing between the University of Edinburgh and HeriotWatt University which is a part of the Edinburgh Research Partnership in Engineering and Mathematics (ERPem). This work was also supported in part by the Natural Sciences and Engineering Research Council (NSERC) of Canada and in part by the Alberta Ingenuity Foundation, Alberta, Canada.

\section{REFERENCES}

[1] Q. Zhao, B. M. Sadler, "A Survey of Dynamic Spectrum Access," IEEE Signal Processing Magazine, vol.24, no. 3, pp. 79-89, May 2007.

[2] I. F. Akyildiz, W. Y. Lee, M. C. Vuran, and S. Mohanty, "NeXt generation/dynamic spectrum access/cognitive radio wireless networks: A survey," Computer Networks, vol. 50, pp.2127-2159, Sept. 2006.

[3] A. Leshem and E. Zehavi, "Bargaining over the interference channel," in Proc. IEEE ISIT, Jul. 2006, Seattle, USA, pp. 2225-2229.

[4] E. Larsson and E. Jorswieck, "Competition versus Collaboration on the MISO Interference Channel", IEEE Journal on Selected Areas in Communications, vol. 26, no. 7, pp. 1059-1069, Sept. 2008.

[5] A. Leshem and E. Zehavi, "Cooperative game theory and the Gaussian interference channel," IEEE Journal on Selected Areas in Communications, vol. 26, no. 7, pp. 1078-1088, Sept. 2008. 
[6] J. Gao, S. A. Vorobyov, and H. Jiang, "Game theoretic solutions for precoding strategies over interference channels," in Proc. IEEE GLOBECOM, New Orleans, USA, Nov. 2008, pp. 1-5.

[7] M. Nokleby, A. L. Swindlehurst, R. Yue and Y. Hua, "Cooperative power scheduling for wireless MIMO networks," in Proc. IEEE GLOBECOM, Washington DC, USA, Nov. 2007, pp. 2982-2986.

[8] S. Ye and R. S. Blum, "Optimized signaling for MIMO interference systems with feedback," IEEE Trans. Signal Processing, vol. 51, no. 11, pp. 2839-2848, Nov. 2003.

[9] E. Telatar, "Capacity of multiantenna Gaussian channels," AT\&T Bell Laboratories, Tech. Memo., Jun. 1995.

[10] W. Wu, W. Rhee, S. Boyd, and J. M. Cioffi, "Iterative water-filling for Gaussian vector multiple-access channels," IEEE Trans. Inform. Theory, vol.50, no. 1, pp.145-152, Jan. 2004.

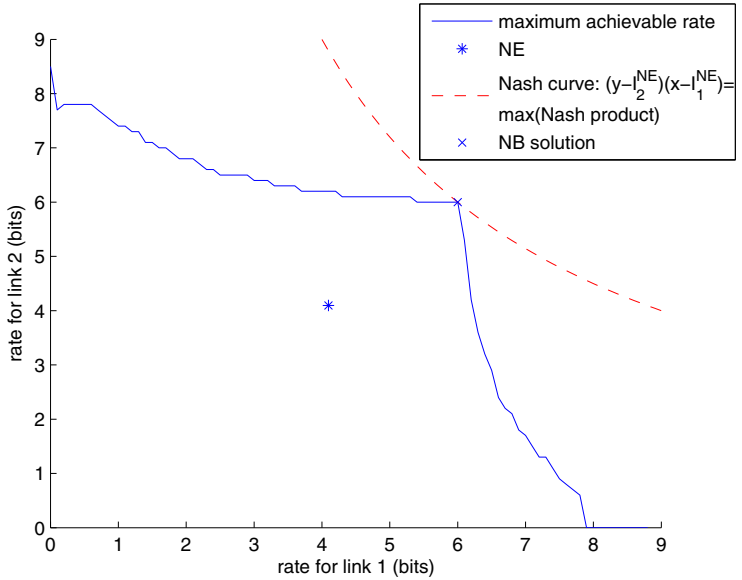

Fig. 1. Maximum achievable rate, NE and NB solution of Type I.

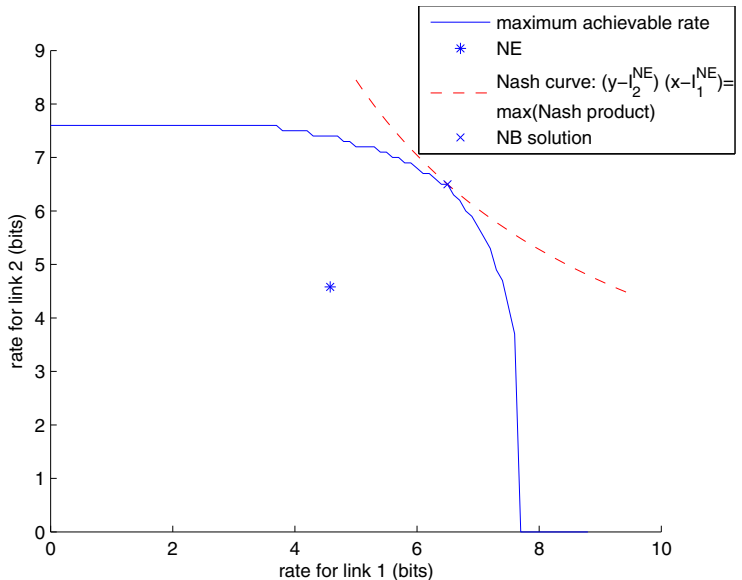

Fig. 2. Maximum achievable rate, NE and NB solution of Type II.

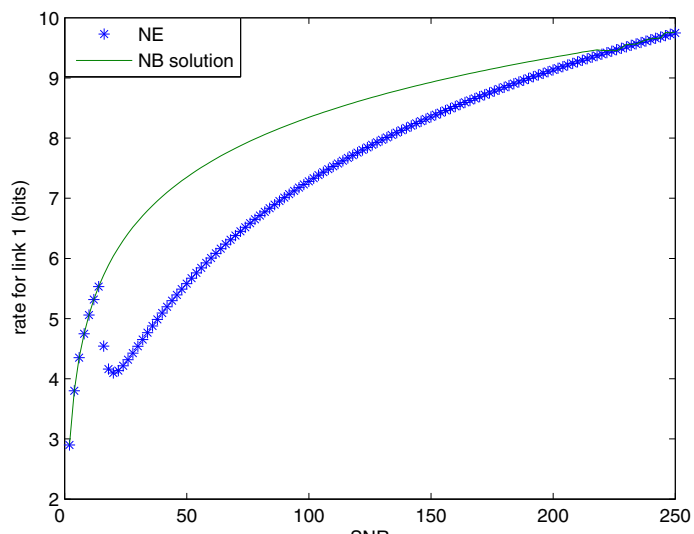

Fig. 3. Impact of SNR on NE and NB solution $(\mathrm{INR}=14)$.
[11] J. F. Nash, "The bargaining problem," Econometrica, vol. 18, no. 2, pp. 155-162, Apr. 1950.

[12] S. Boyd and L. Vandenberghe, Convex Optimization. Cambridge U.K.: Cambridge University Press, 2004.

[13] J. R. Magnus and H. Neudecker, Matrix Differential Calculus with Applications in Statistics and Economics, 2nd ed. New York: Wiley, 1999.

[14] H. Boche, S. Naik and M. Schubert, "Structure of solutions of resource allocation problems under general fairness constraints," in Proc. IEEE ICASSP, Las Vegas, USA, Mar. 2008, pp. 5372-5375.

[15] G. Arslan, M. F. Demirkol, and Y. Song, "Equilibrium efficiency improvement in MIMO interference systems: a decentralized stream control approach," IEEE Trans. on Wireless Communications, vol. 6, no. 8, pp. 2984-2993, Aug. 2007.

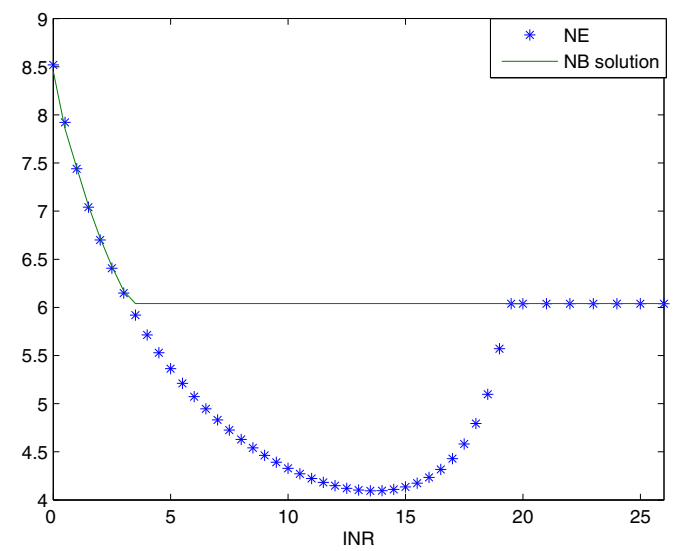

Fig. 4. Impact of INR on NE and NB solution $(\mathrm{SNR}=20)$.

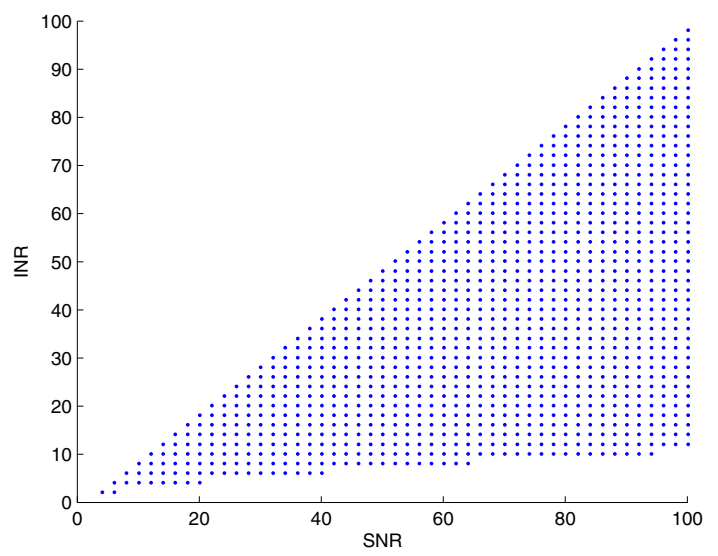

Fig. 5. Existence of NB solution at different SNR and INR.

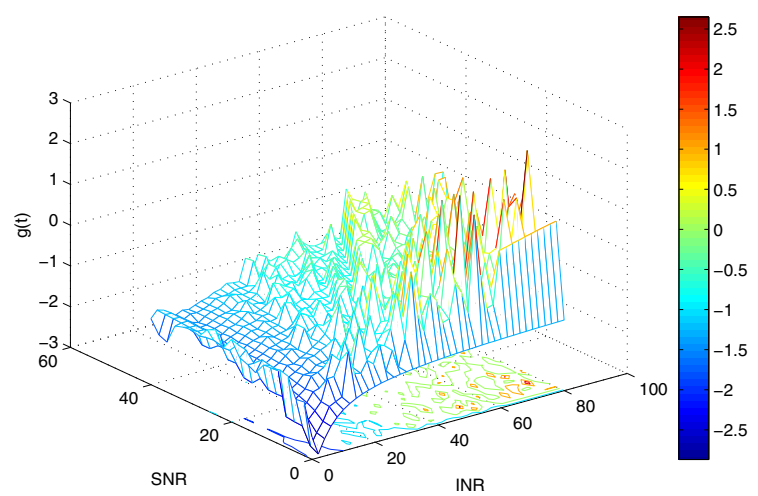

Fig. 6. Concavity of rate product. 